PROCEEDINGS OF THE

AMERICAN MATHEMATICAL SOCIETY

Volume 129, Number 3, Pages 685-693

S 0002-9939(00)05574-X

Article electronically published on April 27, 2000

\title{
SUBGROUP SEPARABILITY, KNOT GROUPS AND GRAPH MANIFOLDS
}

\author{
GRAHAM A. NIBLO AND DANIEL T. WISE
}

(Communicated by Ronald A. Fintushel)

\begin{abstract}
This paper answers a question of Burns, Karrass and Solitar by giving examples of knot and link groups which are not subgroup-separable. For instance, it is shown that the fundamental group of the square knot complement is not subgroup separable. Let $L$ denote the fundamental group of the link consisting of a chain of 4 circles. It is shown that $L$ is not subgroup separable. Furthermore, it is shown that $L$ is a subgroup of every known nonsubgroup separable compact 3-manifold group. It is asked whether all such examples contain $L$.
\end{abstract}

A group $G$ is said to be subgroup separable if each finitely generated subgroup $H$ is the intersection of finite index subgroups of $G$. The first example of a 3-manifold group which is not subgroup separable was given in [BKS]. In that paper, Burns, Karrass, and Solitar observed that their example $K$ is not a knot group, and they asked if knot groups are subgroup separable. The primary purpose of this paper is to answer their question by giving examples of knot and link groups which are not subgroup separable.

In section 1, the example $K$ of $B K S$ is used to show that a closely related group $L$ is not subgroup separable. $L$ is the fundamental group of the complement of a very simple link: the chain of four circles illustrated in Figure 2.

In section $2, L$ plays the role of a poisonous subgroup in the proof of Theorem 2.1 which characterizes the subgroup separability of certain amalgamated free products. For instance, as a special case of Theorem 2.1 we obtain the following corollary:

Corollary. Let $G=\left(F_{m} \times Z_{1}\right) * Z_{2}\left(F_{n} \times Z_{3}\right)$, where for each $i$, we let $Z_{i}$ denote an infinite cyclic group, and we let $F_{i}$ denote a free group of rank $i$. Then $G$ is subgroup separable if and only if $G$ is virtually $F_{q} \times Z_{4}$ for some $q$.

The example of $[\mathrm{BKS}]$ was exploited in $[\mathrm{LN}$ to produce various other nonsubgroup separable groups. For instance, in [LN] the authors construct a nonsubgroup separable example $R *_{Z} R$, where $R$ is the $(4,4,2)$ triangle group. Their examples motivated Theorem 2.1, which places their examples in a more general class.

In section 3, Theorem 2.1 is used to show that various knot groups are not subgroup separable. Perhaps the simplest such example is the following corollary.

Received by the editors April 14, 1998 and, in revised form, May 24, 1999.

2000 Mathematics Subject Classification. Primary 20E26, 20E06, 20F34, 57M05, 57M25.

The second author was supported as an NSF Postdoctoral Fellow under grant no. DMS9627506 . 
3.4 Corollary. The fundamental group of the complement of the square knot is not subgroup separable.

Another source of examples of compact 3-manifold groups which are not subgroup separable is due to $\mathrm{RW}$. Their examples are more geometric than the example of [BKS] and are derived from beautiful ideas involving immersed surfaces that don't satisfy the $k$-plane intersection property.

The groups of both [RW] and [BKS] are studied in [NW] where we show that they contain finitely generated subgroups which are not contained in any proper finite index subgroups, so these groups are as far from being subgroup separable as possible. In section 4 of this paper we show that $L$ is a subgroup of every non-subgroup separable 3-manifold group obtained in [RW], by establishing the following characterization of graph manifolds whose fundamental groups are subgroup separable:

This is dealt with in section 4, where we prove the following:

4.2 Theorem. Let $M$ be a compact graph manifold. Then the following are equivalent:

(1) $\pi_{1} M$ is subgroup separable.

(2) $M$ is a Seifert manifold or $M$ is virtually a torus bundle over $S^{1}$.

(3) There is no embedding of $L$ in $\pi_{1} M$.

In conclusion, the results of this paper show that $L$ occurs as a subgroup of every known non-subgroup separable compact 3-manifold group. It is not difficult to show that every finite index subgroup of $L$ contains a subgroup isomorphic to $L$. This motivates the following question:

Question. Does every non-subgroup separable compact 3-manifold group contain $L$ as a subgroup?

Since $L$ is not a subgroup of any hyperbolic 3-manifold group, the question would be resolved negatively if one could find a hyperbolic 3-manifold whose fundamental group is not subgroup separable [T]. This is an old problem which appears to be quite difficult. We refer the reader to $[\mathrm{W}]$ for a description of some of the latest developments.

\section{The LINK GROUP $L$}

We first recall the example $K$ of [BKS] of a 3-manifold group which is not subgroup separable. It is presented there as $K=\langle y, \alpha, \beta| y^{-1} \alpha y=\alpha \beta, y^{-1} \beta y=$ $\beta\rangle$. It will be convenient to use the following slightly different presentation:

$$
K=\left\langle j, k, t \mid[j, k], t^{-1} j t=k\right\rangle .
$$

An isomorphism between the groups of these two presentations is induced by the map $t \mapsto \alpha, j \mapsto y^{-1}, k \mapsto \beta y^{-1}$.

1.1 Example. Let $L$ denote the group presented by

$$
L=\left\langle x, y, r, s \mid x^{r}=x, y^{r}=y, x^{s}=x\right\rangle .
$$

1.2 Theorem. $L$ is not subgroup separable.

Proof. We prove this by showing that $K$ has an index 2 subgroup $K^{\prime}$ which is isomorphic to a subgroup of $L$. This implies that $L$ is not subgroup separable 


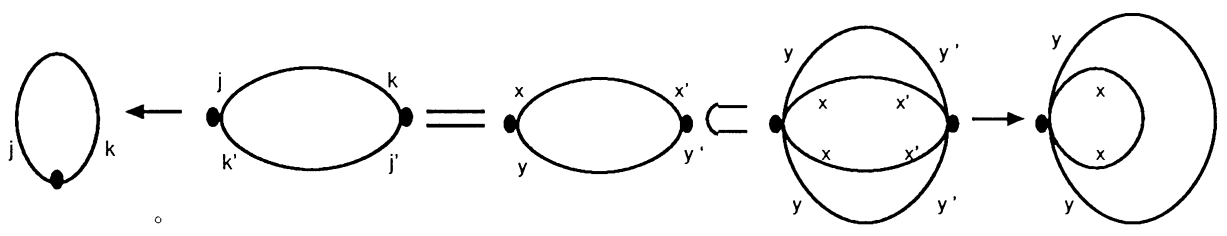

FIGURE 1.

because of the following two easy facts $[\underline{S}]$ : 1) A finite extension of a subgroup separable group is subgroup separable; 2) A subgroup of a subgroup separable group is subgroup separable.

The argument is best explained in terms of graphs of spaces as in Figure 1. Each vertex in Figure 1 represents a torus $S^{1} \times S^{1}$. The two circles in the 1-skeleton of a torus vertex space are oriented and labeled by the two distinct symbols incident at the corresponding vertex.

Each edge in Figure 1 represents a cylinder $S^{1} \times I$ where the boundary circles are oriented consistently and labeled as indicated in the diagram. Each such cylinder edge space is attached at each end by a label preserving and orientation preserving combinatorial map.

The maps between the graphs in Figure 1 are described as follows: The map designated by the equality sign is the obvious isomorphism. The map designated by the inclusion sign is the obvious label preserving inclusion. The two maps designated by arrows are double covers which preserve only the alphabetical part of the label, e.g., both $j$ and $j^{\prime}$ map to $j$.

Furthermore, our maps between two graphs of spaces are the obvious combinatorial maps corresponding to the maps between their underlying graphs.

It is easy to see that the 2-complexes on the left and right are isomorphic to the standard 2-complexes of the presentations of $K$ and $L$ respectively. We let $K^{\prime}$ denote $\pi_{1}$ of the second 2-complex (from the left), and since the map to the leftmost complex is a double cover, $K^{\prime}$ is of index 2 in $K$.

To show that $K^{\prime}$ is isomorphic to a subgroup of $L$, we observe that the sequence of maps from the second complex to the fifth complex induces $\pi_{1}$-injections since they are respectively an isomorphism, a subgraph of groups, and a double cover.

1.3 Theorem. The group $L$ is isomorphic to the fundamental group of the complement of the link illustrated in Figure 2.

Proof. We consider the Wirtinger presentation of the link diagram in Figure 2. Using the oriented generators labeling the overpasses we obtain the presentation

$$
\left\langle a, b, c, d, e, f \mid \begin{array}{l}
b a=a d, \quad c d=d e, \quad f e=e f \\
a b=b a, \quad d c=c b, \quad \text { ef }=f c
\end{array}\right\rangle .
$$

We first remove the relation $d c=c b$. Now observe that because $a b=b a$, the relation $b a=a d$ is equivalent to the relation $b=d$. Likewise, because $f e=e f$, the relation $e f=f c$ is equivalent to $e=c$. So we obtain the following simpler presentation:

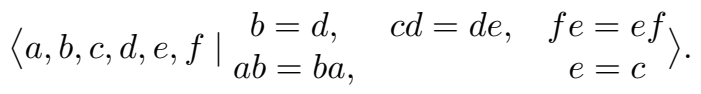




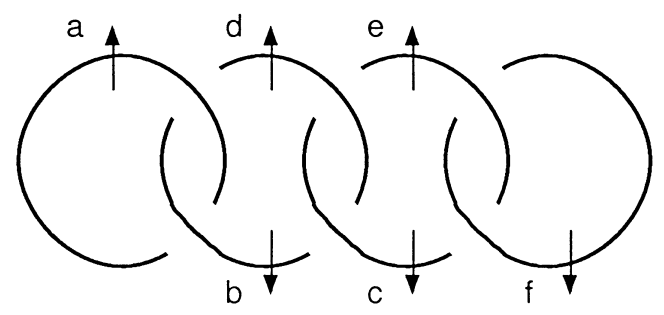

FiguRE 2.

Next we remove the generator $d$ and the relation $b=d$, and we remove the generator $c$ and the relation $e=c$, to obtain:

$$
\left\langle a, b, e,\left.f\right|_{a b=b a,} \quad e b=b e, \quad f e=e f\right\rangle .
$$

The resulting presentation is obviously isomorphic to the presentation for $L$ given above, using the map $a \mapsto s, b \mapsto x, e \mapsto r, f \mapsto y$.

1.4 Theorem. $L$ is a subgroup of $K$.

Proof. Consider the homomorphism $K \rightarrow Z$ induced by $j \mapsto 0, k \mapsto 0, t \mapsto 1$. Examination of the covering space shows that the kernel has presentation $\left\langle j_{i}, k_{i}\right|$ $\left.\left[j_{i}, k_{i}\right], j_{i}=k_{i+1}(i \in Z)\right\rangle$ which is equivalent to $\left\langle j_{i} \mid\left[j_{i}, j_{i+1}\right](i \in Z)\right\rangle$. The group $L$ is obviously a subgroup of this kernel.

\section{Amalgamated free products}

2.1 Theorem. Consider a non-trivial amalgamated free product of the form $G=$ $A *_{E} B$, where each of $A$ and $B$ is virtually the direct product of a free group and an infinite cyclic group, and $E$ is virtually cyclic. Then the following are equivalent:

(1) $G$ is subgroup separable.

(2) $G$ is virtually $F_{q} \times Z$.

(3) E has a cyclic subgroup of finite index which is normal in $A$ and $B$ (and thus $G)$.

(4) $L$ is not a subgroup of $G$.

Before proceeding with the proof of Theorem 2.1, we mention the following generalization:

2.2 Remark: Graphs of groups. One can show similarly that a finite graph of groups with virtually $F_{m} \times Z$ vertex groups and virtually cyclic edge groups is subgroup separable if and only if it is virtually $F_{q} \times Z$. The proof is similar, except that in this case one also uses the fact that if the group is subgroup separable, then there is no relation of the form $\left(x^{n}\right)^{g}=x^{m}$ where $x$ has infinite order and $n \neq \pm m$.

$(3) \Rightarrow(2)$. Let $N$ denote a cyclic subgroup of finite index of $E$ which is normal in $A$ and $B$. Consider the exact sequence

$$
1 \rightarrow N \rightarrow A *_{E} B \rightarrow A / N *_{E / N} B / N \rightarrow 1 .
$$

Since $A$ is virtually $F_{n} \times Z, A / N$ is virtually free because $\left(F_{n} \times Z\right) /\left(\left(F_{n} \times Z\right) \cap N\right)$ is virtually free, and it is of finite index in $A / N$. Similarly, $B / N$ is virtually free and $E / N$ is finite and so we see that $G / N$ is virtually free. 
Let $F$ denote a free subgroup of finite index in $G / N$, and let $J$ denote its preimage in $G$. Then we have a short exact sequence

$$
1 \rightarrow N \cap J \rightarrow J \rightarrow F
$$

Since $F$ is free, we can express $J$ as a semidirect product $J=(N \cap J) \ltimes F$.

Since $N \cap J$ is cyclic, there is a subgroup $F^{\prime}$ of index $\leq 2$ in $F$ which centralizes $N \cap J$. Thus $(N \cap J) \times F^{\prime} \subset(N \cap J) \ltimes F \subset G$ is the desired direct product subgroup.

$(2) \Rightarrow(1)$. It is sufficient to show that $F_{2} \times Z$ is subgroup separable, and this was proven in $\mathrm{AG}$ ] or $\left[\mathrm{S}\right.$. Alternately, the group $\left\langle a, b \mid a^{3}=b^{3}\right\rangle$ is subgroup separable by the result of [BBS], and it is easily seen to contain $F_{2} \times Z$ as a subgroup. Indeed, this follows from $(3) \Rightarrow(2)$.

$(1) \Rightarrow(4)$. A subgroup of a subgroup separable group is subgroup separable, but $L$ is not subgroup separable by theorem 1.2.

$(4) \Rightarrow(3)$. We will argue by contradiction. Without loss of generality we assume that $E$ does not have a cyclic subgroup of finite index which is normal in $A$.

By hypothesis, $A$ is virtually $F_{n} \times Z$, and now the argument breaks down into cases according to the value of $n$. We need not consider the case $n=0$ because we have assumed that $E$ does not have a cyclic subgroup of finite index which is normal in $A$. In case $n=1$ we will show that the group $K$ embeds in $A *_{E} B$, and so it is not subgroup separable. In case $n \geq 2$, we will show that the group $L$ embeds in $A *_{E} B$ and so it is not subgroup separable.

Let $c$ generate an infinite cyclic subgroup of $E$. We will use the fact that there exists $m>0$ such that $\left\langle c^{m}\right\rangle$ is normalized by some element $b \in B-E$. This is easy to prove by using the fact that $E$ is a proper subgroup of $B$, and that $B$ is virtually $F_{m} \times Z$.

In case $n=1$, choose a normal subgroup of finite index $T \subset A$ such that $T \cong Z \times Z$, and $T \cap E \subset\left\langle c^{m}\right\rangle$. Let $e$ denote a generator of $T \cap E$. By hypothesis, $T \cap E$ is not normal in $A$ and so we may choose an element $a \in A$ such that $e^{a} \notin T \cap E$. Observe that $e^{b a}$ and $e$ form a basis for a rank 2 subgroup of $T$. If we think of $K$ as an HNN extension with stable letter $t$, then it follows from the normal form theorem that $K$ embeds in $G$ using the homomorphism induced by $j \mapsto e, k \mapsto e^{b a}, t \mapsto b a$. Since, by Theorem 1.4, $L$ embeds in $K$, we see that $L$ embeds in $G$.

In case $n>1$ then, without loss of generality, we choose a subgroup of finite index $F_{n} \times Z$ of $A$, such that $\left(F_{n} \times Z\right) \cap E$ is contained in $\left\langle c^{m}\right\rangle$. Let $\left(F_{n} \times Z\right) \cap E$ be generated by $(w, z) \in F_{n} \times Z$. Observe that $w \in F_{n}$ is not trivial, because if it were trivial, then $\left(F_{n} \times Z\right) \cap E$ would be a subgroup of finite index of the center of $F_{n} \times Z$, and thus normal in $A$.

Choose an element $(u, z) \in F_{n} \times Z$ such that $(u, z)$ and $(w, z)$ form a basis for a free group of rank 2.

If we think of $L$ as an HNN extension with stable letter $s$, then it follows from the normal form theorem that $L$ embeds in $G$ using the map $x \mapsto(w, z), y \mapsto$ $(u, z), r \mapsto\left(1_{F_{n}}, 1\right), s \mapsto b\left(1_{F_{n}}, 1\right) b^{-1}$. 


\section{KNOT GROUPS THAT AREN'T SUBGROUP SEPARABLE}

We will need the following result in order to apply Theorem 2.1 to the case of the sum of torus knots in Theorem 3.2 below.

3.1 Lemma. Let $T=T_{p, q}$ be a non-trivial torus knot complement, and let $m$ denote a meridianal curve in $T$. Then $\langle m\rangle$ does not have a subgroup of finite index which is normal in $\pi_{1} T$.

Proof. $\pi_{1} T$ can be presented as $\left\langle a, b \mid a^{p}=b^{q}\right\rangle$, where, because $T$ is non-trivial, we may assume that $p>q>1$ and $\operatorname{GCD}(p, q)=1$. By Proposition 3.28 of [BZ], the meridianal curve is represented by the element $m=a^{u} b^{v}$ where $p v+q u=1$.

Now, consider the homomorphism

$$
\left\langle a, b \mid a^{p} b^{-q}\right\rangle \rightarrow\left\langle\bar{a}, \bar{b} \mid \bar{a}^{p}, \bar{b}^{q}\right\rangle .
$$

Observe that since $p>q>1$, the image group is virtually free of rank $\geq 2$, so there are no normal infinite cyclic subgroups. Note that it follows from $p v+q u=1$ that $p \nmid u$ and $q \backslash \gamma$. Consequently, both $\bar{a}^{u}$ and $\bar{b}^{v}$ are non-trivial, and so, by the normal form theorem, the image $\bar{a}^{u} \bar{b}^{v}$ of $m$ has infinite order. Consequently no power of the image of $m$ generates a normal subgroup in the quotient, and therefore no power of $m$ generates a normal subgroup in $\pi_{1} T$.

3.2 Theorem. The sum of two non-trivial torus knots is not subgroup-separable.

Proof. Let $T$ and $T^{\prime}$ denote the torus knot complements. Let $m$ and $m^{\prime}$ denote elements of $\pi_{1} T$ and $\pi_{1} T^{\prime}$ represented by appropriate meridianal curves of $T$ and $T^{\prime}$ respectively. Now the fundamental group of the connected sum $T \# T^{\prime}$ is isomorphic to $\pi_{1} T *_{m=m^{\prime}} \pi_{1} T^{\prime}$. Since a torus knot group is virtually $F_{n} \times Z$ (from Theorem 2.1 above or [BZ]), it follows from Theorem 2.1 that $\pi_{1}\left(T \# T^{\prime}\right)$ is subgroup separable if and only if $\langle m\rangle$ and $\left\langle m^{\prime}\right\rangle$ have subgroups of finite index which are normal in $T$ and $T^{\prime}$ respectively. But by Lemma 3.1 this is true only if $T$ and $T^{\prime}$ are trivial links.

3.3 Remark: Other connected sums. In fact, a similar argument shows that the connected sum of a non-trivial torus knot and any non-trivial knot has a non subgroupseparable $\pi_{1}$.

Since the square knot is the connected sum of two trefoil knots, we obtain:

3.4 Corollary. The fundamental group of the square knot complement is not subgroup separable.

\section{GRAPH MANIFOLDS}

We refer the reader to $[\mathrm{RW}$ and the references therein for information about Seifert manifolds and graph manifolds.

Examples of graph manifolds whose fundamental groups aren't subgroup separable are due to $[\mathrm{RW}$. The point of the following theorem is that the failure of the subgroup separability in this case can be linked to the presence of a subgroup isomorphic to $L$, where $L$ is the link group of section 1 .

4.1 Lemma. Consider the group $G=\left(F_{m} \times Z\right) *_{T}\left(F_{n} \times Z\right)$, where $n, m \geq 2$, and where the amalgamated subgroup $T$ is a maximal $Z \times Z$ subgroup in each factor. Suppose that the embedding of the amalgamated subgroup does not identify the centers of the factors. Then there is an embedding $L \rightarrow G$. 
Proof. Without loss of generality, suppose that the non-central element $(w, z)$ of the left factor $F_{m} \times Z$ is identified with the generator $\left(1_{F}, 1\right)$ of the center of the right factor $F_{n} \times Z$.

Now, using the fact that $m \geq 2$, we can choose a subgroup $R \subset F_{m} \times Z$ such that $R \cong Z \times Z$ and $R \cap T=\left\langle\left(1_{F}, 1\right)\right\rangle$. Finally, observe that because of the normal form theorem, the obvious map $\left(F_{m} \times Z\right) *_{(w, z)=\left(1_{F}, 1\right)} R \rightarrow\left(F_{m} \times Z\right) *_{T}\left(F_{n} \times Z\right)$ is an inclusion. But $\left(F_{m} \times Z\right) *(w, z)=\left(1_{F}, 1\right) R$ satisfies the hypothesis of Theorem 2.1 and thus contains $L$ as a subgroup, and consequently so does $\left(F_{m} \times Z\right) *_{T}\left(F_{n} \times Z\right)$.

4.2 Theorem. Let $M$ be a compact graph manifold. Then the following are equivalent:

(1) $\pi_{1} M$ is subgroup separable.

(2) $M$ is a Seifert manifold, or $M$ is a torus bundle over $S^{1}$, or $M$ is the union of two twisted I-bundles over the Klein bottle, glued together along their boundaries.

(3) There is no embedding of $L$ in $\pi_{1} M$.

$(2) \Rightarrow(1)$. The fact that the fundamental group of a Seifert fibered 3-manifold is subgroup separable was proved by Scott in $[\underline{S}]$. For a torus bundle over $S^{1}$ we first observe that its fundamental group $G$ is a semidirect product $A \rtimes N$, where $A$ is isomorphic to $\mathbb{Z} \times \mathbb{Z}$ and $N$ is isomorphic to $\mathbb{Z}$. Therefore $G$ is polycyclic, and so $G$ is subgroup separable because Malćev proved that every polycyclic group is subgroup separable (see 5.4 .16 of [Rob]). If $M$ is a union of two twisted $I$-bundles over the Klein bottle, then it is double covered by a torus bundle over $S^{1}$, and we are reduced to the previous case, since finite extensions of subgroup separable groups are themselves subgroup separable.

$(1) \Rightarrow(3)$. By Theorem 1.2 above, $L$ is not subgroup separable, and therefore $\pi_{1} M$ is not subgroup separable.

$(3) \Rightarrow(2)$. Suppose that $M$ is a graph manifold and there is no embedding of $L$ in $\pi_{1}(M)$. Without loss of generality we may assume that $M$ is decomposed minimally as a graph manifold, i.e., that no two adjacent vertex submanifolds have a union which may be Seifert fibered.

If the underlying graph has terminal vertices which are fibered over a flat orbifold, then we may take a double cover of the manifold in which each of them is covered by the direct product of a circle and an annulus. The remaining components each lift to two components of degree one. The underlying graph of the double cover now has vertices isomorphic to $T^{2} \times I$, one for each of the original terminal vertices, and these all have valency two, so are replaced by edges in a minimal decomposition. We introduce no new terminal vertices in this process, so the double cover has no terminal vertices fibered over a flat orbifold.

Now suppose that the double cover is either a torus bundle over $S^{1}$ or a Seifert fibered space. In the first case it is easy to see that either $M$ is itself a torus bundle, or it is double covered by a torus bundle and is a union of two twisted $I$-bundles over the Klein bottle. In the case when the double cover is Seifert fibered it is easy to see that $\pi_{1}(M)$ contains a central, infinite cyclic subgroup and we may appeal to Gabai Casson Jungreis, wherein it is shown that any compact irreducible 3 -manifold whose fundamental group contains a normal, infinite cyclic subgroup is Seifert fibered. Hence it is sufficient to show that the double cover is indeed either a Seifert fiber space or a torus bundle. 
Recall that we are now assuming that the minimal graph decomposition we are using for our manifold has no terminal vertices whose base space is a flat orbifold. We will consider first the case when the minimal graph decomposition has at least two vertex manifolds. In this case let $M_{1}, M_{2}$ be adjacent vertex manifolds in the minimal decomposition. Let $M^{\prime}$ denote the graph manifold obtained by glueing $M_{1}$ and $M_{2}$ along a single torus boundary component so that the glueing agrees with the corresponding identification in $M$. The result is homeomorphic to an incompressible submanifold of $M$, so its fundamental group injects in $\pi_{1}(M)$. In particular there is no embedding of $L$ in $\pi_{1}\left(M^{\prime}\right)$.

Since the graph decomposition of $M$ is minimal, neither component, $M_{1}$ nor $M_{2}$, is homeomorphic to $T^{2} \times I$, and since it has no terminal vertex spaces fibered over a flat orbifold, neither is fibered over any flat orbifold. It follows that both are Seifert fibered over a surface of negative Euler characteristic, and so each $M_{i}$ has a finite cover which is homeomorphic to a product $M_{i}^{\prime}=\Sigma_{i} \times S^{1}, i=1,2$, where $\Sigma_{i}^{\prime}$ is also a surface of negative Euler characteristic, and which contains a degree 1 component of the pre-image of the identified torus. A standard argument shows that glueing the two manifolds $M_{i}^{\prime}$ along this lift, respecting the glueing induced from $M^{\prime}$, gives a manifold $M^{\prime \prime}$ homeomorphic to a compact core for a cover of $M^{\prime}$. In particular $\pi_{1}\left(M^{\prime \prime}\right)$ contains no copy of $L$. But $\pi_{1}\left(M^{\prime \prime}\right)$ is an amalgamated free product of two products $F_{i} \times Z$, where $F_{i}=\pi_{1}\left(\Sigma_{i}^{\prime}\right)$ is free of rank at least 2 . Now by Lemma 4.1, we see that the identification of the two boundary tori in $M^{\prime \prime}$ must identify the centers of $\pi_{1}\left(M_{1}^{\prime}\right)$ and $\pi_{1}\left(M_{2}^{\prime}\right)$, which are the infinite cyclic subgroups carried by the fibers of $M^{\prime \prime}$. Since these are the lifts of the fibers in $M^{\prime}$, it also follows that the glueing of the boundary tori in $M^{\prime}$ identifies the normal cyclic subgroup carried by its fibers so we can homotop the glueing map to identify the fibers to see that $M^{\prime}$ may itself be Seifert fibered. But this contradicts the fact that $M^{\prime}$ is a union of two vertex manifolds in a minimal graph decomposition of $M$, so the minimal graph decomposition of $M$ must have had only one vertex.

Now suppose that a minimal graph decomposition of $M$ has only one vertex and $n \geq 2$ edges. In this case the vertex manifold has at least four boundary components, and so it has a finite cover homeomorphic to a product $\Sigma \times S^{1}$ where $\Sigma$ is a surface of strictly negative Euler characteristic, so its fundamental group is $F_{n} \times Z$ for some $n \geq 2$. Now we can construct a double cover of $M$ by splitting it open along one of the non-separating tori, and glueing two copies of the result along their boundaries to respect the original identifications. The resulting manifold $M^{\prime}$ has a graph manifold decomposition with two vertices and $2 n$ edges, and it is not too hard to see that this is minimal. It now follows from the previous case that $\pi_{1}\left(M^{\prime}\right)$ must contain $L$ as a subgroup.

Finally there are two cases to consider: when the minimal graph decomposition of $M$ consists of a single vertex manifold and no edge manifolds, in which case $M$ is Seifert fibered, and when the minimal graph decomposition of $M$ consists of a single vertex manifold homeomorphic to $T^{2} \times I$, and a single edge manifold $T^{2} \times I$. In this latter case $M$ is a torus bundle over $S^{1}$, as required.

\section{ACKnowledgments}

We wish to thank John Meier for a conversation that stimulated this work. We are also grateful to John Stallings, Walter Neumann, and the referee for helpful comments. 


\section{REFERENCES}

[AG] R.B.J.T. Allenby and R.J. Gregorac, On locally extended residually finite groups, Conference on Group Theory (Univ. Wisconsin-Parkside, Kenosha, Wis., 1972). Lecture Notes in Math., Vol. 319, Springer, Berlin, 9-17. MR 52:3333

[BBS] A.M. Brunner, R.G. Burns, and D. Solitar, The subgroup separability of free products of two free groups with cyclic amalgamation, Contemp. Math. 33 (1984), 90-115. MR 86e:20033

[BKS] R.G. Burns, A. Karrass, and D. Solitar, A note on groups with separable finitely generated subgroups, Bull. Aust. Math. Soc. 36 (1987), 153-160. MR 88g:20057

[BZ] G. Burde and H. Zieschang, Knots, Walter de Gruyter, Berlin - NY, 1985. MR 87b:57004

[CJ] A. Casson and D. Jungreis, Convergence groups and Seifert fibered 3-manifolds, Invent. Math. 118 (1994), 441-456. MR 96f:57011

[G] D. Gabai, Convergence groups are Fuchsian groups, Ann. of Math. 136 (1992), 447-510. MR 93m:20065

[LN] D.D. Long and G.A. Niblo, Subgroup Separability and 3-manifold groups, Math. Z. 207 (1991), 209-215. MR 92g:20047

[LS] R.C. Lyndon, and P.E. Schupp, Combinatorial Group Theory, Springer-Verlag, 1977. MR 58:28182

[NW] G.A. Niblo and D.T. Wise, The engulfing property for 3-manifolds, in: The Epstein birthday schrift (1998), 413-418. MR 99k:57041

[Rob] D.J.S. Robinson, A Course in the Theory of Groups (Graduate Texts in Math., vol 80), Springer-Verlag, 1993. MR 94m:20001

[Rol] D. Rolfson, Knots and Links, Publish or Perish, Inc., Houston TX, 1990.

[RW] J.H. Rubinstein and S. Wang, On $\pi_{1}$-injective surfaces in graph manifolds, Comm. Math. Helv. 73 (1998), 499-515. MR 99h:57039

[S] P. Scott, Subgroups of surface groups are almost geometric, J. London Math. Soc. (2) 17 (1979), 555-565. MR 58:12996 correction MR 87k:57003

[T] W. Thurston, Three dimensional manifolds, Kleinian groups and hyperbolic geometry, Bull. Amer. Math. Soc. 6 (1982), 357-382. MR 83h:57019

[W] D.T. Wise, Subgroup separability of the figure 8 knot group, Preprint (1998).

Faculty of Mathematical Studies, University of Southampton, Highfield, SouthampTON, SO17 1BJ, ENGLAND

E-mail address: gan@maths.soton.ac.uk

Department of Mathematics, University of California at Berkeley, Berkeley, CalIFORNIA 94720

Current address: Department of Mathematics, White Hall, Cornell University, Ithaca, New York 14853

E-mail address: daniwise@math.cornell.edu 